\title{
Bimorph X-ray optics for time-resolved experiments
}

Anton Kulikov $^{1}$, Alexander Blagov ${ }^{2}$, Nikita Marchenkov ${ }^{2}$, Anton Targonsky ${ }^{1}$, Yuriy Pisarevsky ${ }^{1}$, Mikhail Kovalchuk ${ }^{2}$

${ }^{1}$ FSRC Crystallography And Photonics RAS, Moscow, Russian Federation, ${ }^{2}$ NRC Kurchatov Institute, Moscow, Russian Federation E-mail: imegalomanius@gmail.com

One of the most significant scientific interests for today is the development of research approaches for the dynamics investigation of processes occurring in the samples, particularly investigation of the material properties in terms of external influences. The key point for such researches is the speed of experiment parameters controlling, i.e. high temporal resolution.

The mechanical systems commonly used for this purpose produce insufficient performance in terms of temporal resolution. As a replacement for mechanical systems, the adaptive-controlled X-ray optical elements based on resonators with longitudinal acoustic wave modulation were developed in our laboratory of X-ray methods of analysis [1]. However, their application is limited due to the narrow range of the lattice parameter variation and the angular position of $X$-ray beam correspondingly. Another approach involves the piezoelectric ceramic materials characterized by low latency and high efficiency. However, their major drawback is caused by hysteresis-dependent polarizability within external electric field, which affects the accuracy and positioning time of X-ray beam.

In this work, the hysteresis-free electrically driven element based on monolithic bimorph piezoactuator was first proposed and successfully tested. Lithium niobate (LiNbO3) actuator crystal consists of two layers with opposite polarization directions and operates on the bending principle [2], which can significantly increase the angular adjustment range. Elements were developed by the Moscow Institute of Steel and Alloys in cooperation with our laboratory.

For the experiments the laboratory source based on X-Ray tube with molybdenum anode was used. A thin Si plate was attached to the bimorph bending element edge to satisfy Bragg or Laue geometry. The electronically controlled adjustment of element's angular position was implemented within the applied constant electric field with the range of up to hundred arcseconds in static operation mode. Angular rearrangement of bimorph element is characterized by a strict linear shift of the rocking curve, reproducibility and relatively low control voltage [3].

The next step was a successfully implemented resonant operating mode of the composite Si+bimorph element as an adaptive monochromator. This mode allows to reach the larger angular amplitude of the Si crystal that was up to thousand arcseconds at resonant frequency and harmonic signal amplitude of 50V. It allowed to carry out the energy scan in wavelength range for the lines $\mathrm{Ka} 1$ - Ka2 of laboratory source. For the X-ray tube spectrum recording a multichannel analyzer was used. It splits the period of applied signal into channels and thereby allows one to accumulate intensity on the detector as a function of the signal phase. Each phase corresponds to the certain orientation angle of the monochromator and hence a certain emission wavelength.

The above technique with Si+bimorph element as monochromator was used to accomplish the double-axis diffractometry method with time resolution to separate fast piezoelectric effect and charge drain processes of dielectric crystals such as Li2B4O7 in external DC field.

Thus, using a high brilliance synchrotron source one can implement the energy scan in white beam or measure rocking curves of the sample in divergent monochromatic beam with time resolution of up to milliseconds.

[1] Kovalchuk M.V. et al. (2011) Crystallography Reports, V.56, №5, pp.828-831.

[2] Bykov A.S. et al. (2014) Russian microelectronics, V.43, №8, pp.536-542.

[3] Blagov A.E. et al. (2016) Instruments and Experimental Techniques, V.59, №5, pp.728-732.

Keywords: bimorph actuator, time resolution, $x$-ray diffraction 